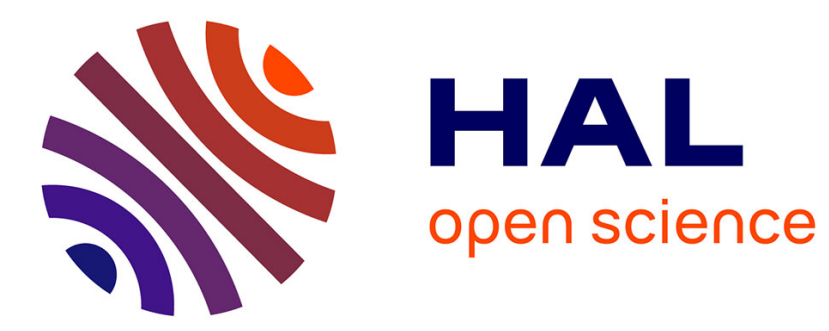

\title{
Propulsion by reciprocal motion into granular media
}

Baptiste Darbois Texier, Alejandro Ibarra, Francisco Melo

\section{To cite this version:}

Baptiste Darbois Texier, Alejandro Ibarra, Francisco Melo. Propulsion by reciprocal motion into granular media. Physical Review Fluids, 2021, 6, 10.1103/physrevfluids.6.034604 . hal-03498761

\section{HAL Id: hal-03498761 https://hal.science/hal-03498761}

Submitted on 21 Dec 2021

HAL is a multi-disciplinary open access archive for the deposit and dissemination of scientific research documents, whether they are published or not. The documents may come from teaching and research institutions in France or abroad, or from public or private research centers.
L'archive ouverte pluridisciplinaire HAL, est destinée au dépôt et à la diffusion de documents scientifiques de niveau recherche, publiés ou non, émanant des établissements d'enseignement et de recherche français ou étrangers, des laboratoires publics ou privés. 


\title{
Propulsion by reciprocal motion into granular media
}

\author{
Baptiste Darbois Texier $\odot,{ }^{1,2, *}$ Alejandro Ibarra, ${ }^{2}$ and Francisco Melo ${ }^{2}$ \\ ${ }^{1}$ Université Paris-Saclay, CNRS, FAST, 91405 Orsay, France \\ ${ }^{2}$ Departamento de Física Universidad de Santiago de Chile, Avenida Ecuador 3493, 9170124 Estación \\ Central, Santiago, Chile Center for Soft Matter Research, SMAT-C, Avenida Bernardo O'Higgins 3363, \\ Estación Central, Santiago, Chile
}

(Received 18 November 2019; accepted 16 November 2020; published 5 March 2021)

\begin{abstract}
The force experienced by an object moving in a granular medium is a necessary input to describe locomotion problems in such a context. Towards this objective, resistive force theory (RFT) has been developed for granular flows inspired by previous developments in the case of viscous flows. In viscous fluids, a reciprocal motion does not lead to a net propulsion due to the kinematics reversibility of viscous flows that is included in the RFT. We show that, in a granular medium, a reciprocal motion allows for propulsion. We investigate the specific mechanisms underlying this propulsion and discuss how they differ from that of viscous flows. Understanding these phenomena permit both the accurate description of locomotion in sand and the refinement of the RFT for granular materials. These achievements provide opportunities towards the development of robots that are able to progress inside granular media and are suitable for controlling industrial processes.
\end{abstract}

DOI: 10.1103/PhysRevFluids.6.034604

\section{INTRODUCTION}

Surviving in deserts requires the ability to manage low water and food disponibility, few natural habitats, violent sand storms, and elevated surface temperatures. An elegant strategy employed by desert animals to overcome these challenges is to burrow and swim below the sand surface. The best known example is the sandfish lizard that undulates its body reaching speeds of two body lengths per second [1,2]. This mode of locomotion has inspired biomimetic robots that operate in unconsolidated soils [3] and are suitable for rescue missions and exploration of industrial facilities [4]. Much less investigated are desert beetles that use a completely different strategy to propel below the sand surface [5-7]. These animals activate their legs incorporating a horizontal periodic motion. For a quantitative description of the propulsion of these animals and robots it is essential to understand frictional forces affecting the dynamics of an object in granular materials. In recent years, significant efforts have been made to rationalize these forces, especially through resistive force theory (RFT) originally developed in the context of viscous flows [8]. This theory accounts for the force experienced by a segment into a granular medium by means of a Coulombian-like friction and an experimental calibration of the friction coefficients and by neglecting long-range interactions between segments. RFT predicts successfully the propulsion of sandfish lizards in sand [9], the dynamics of small legged robots' locomotion on granular substrates [10], and the force experienced by a plate dragged into flowable materials [11]. Moreover, Askari et al. show that granular RFT arises from two of the most salient mechanical features of dry granular media: a frictional yield criterion and the absence of cohesion [12]. However, the RTF does not capture the propulsion by reciprocal motion as employed by desert beetles.

\footnotetext{
*baptiste.darbois-texier@universite-paris-saclay.fr
} 
(a)

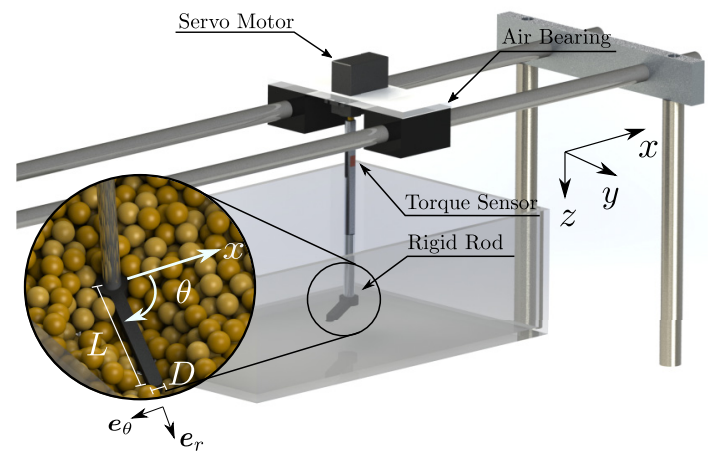

(b)

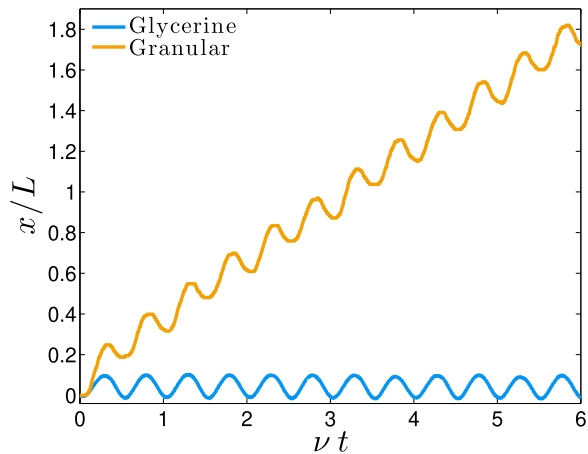

FIG. 1. (a) The experimental setup. A rod with a length of $L=60 \mathrm{~mm}$ and a diameter of $D=6 \mathrm{~mm}$ oscillates according $\theta(t)=\theta_{m} \sin (2 \pi v t)$ with respect to the $x$-direction. (b) Position of the rod's head along the $x$-direction as a function of time in glycerine (blue solid line) and in a granular medium (orange solid line) for an angular amplitude $\theta_{m}=45^{\circ}$, frequency $v=0.26 \mathrm{~Hz}$, and depth $h=40 \mathrm{~mm}$.

In viscous fluids at low Reynolds numbers, propulsive motions are associated with Purcell's scallop theorem, which states that a reciprocal motion of an object cannot lead to a net displacement $[13,14]$ due to the symmetry of the viscous flow to time inversion. The darkling beetle, when in sand, propels by a reciprocal motion in the legs (perfectly symmetric time cycle), which indicates that the granular flow is not reciprocal and that the friction properties of the medium are modified by the passage of the object. As a consequence, the scallop theorem does not hold in granular materials and the RFT should be refined. The breakdown of the scallop theorem has been studied in viscoelastic fluids [15], shear-thinning and shear-thickening fluids when time-asymmetric stroke patterns are employed [16], and for a swarm of reciprocal microswimmers [17]. However, the failure of the scallop theorem in granular materials is not due to these phenomena and remains unexplored. In this article, we study the behavior of a rod oscillating horizontally in a particulate material and explore its efficiency as a propulsive mechanism. The specificities of granular flows that are responsible for the scallop theorem breakdown are addressed, and empirical laws are proposed to include these effects into the RFT.

\section{EXPERIMENTS}

Our setup is illustrated in Fig. 1(a). A pivoting rigid $\operatorname{rod}(L=60 \mathrm{~mm}$ and $D=6 \mathrm{~mm})$ is entered into a rectangular box filled with plastic grains of mean diameter $d=2 \mathrm{~mm}$, bulk density $\rho=$ $1540 \mathrm{~kg} / \mathrm{m}^{3}$, and friction coefficient $\mu=0.61 \pm 0.01$. A sufficiently large box $(40 \times 30 \times 20 \mathrm{~cm})$ has been chosen to prevent the influence of the edges and Janssen's effect [18]. An external motor is connected to an extremity of the rod to impose the angular motion in the horizontal plane $(x, y)$ at depth $h$ below the granular surface. The angular position of the rod is varied sinusoidally with a frequency $v$ and a maximal angle $\theta_{m}$ according to $\theta(t)=\theta_{m} \sin (2 \pi v t)$ with respect to the $x$ direction. The whole system, composed of the external motor and the immersed rod, is constrained along the $x$-axis by a low friction air-pressurized guide. The rod dimensions are chosen to have similar aspect ratios $L / D$ and $D / d$ as for a beetle leg in sand. Also in the experiments, we prescribe small rod velocity $(L v<5 \mathrm{~cm} / \mathrm{s})$ to ensure a quasistatic regime of granular flow as is the case for beetle locomotion within sand. These choices guarantee that our setup constitutes a scaled-up physical model of beetle subsurface motion. 
(a)

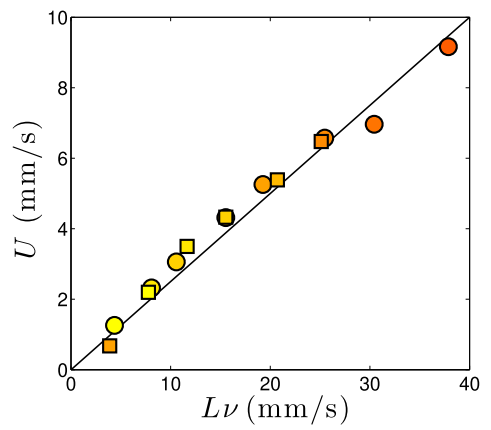

(b)

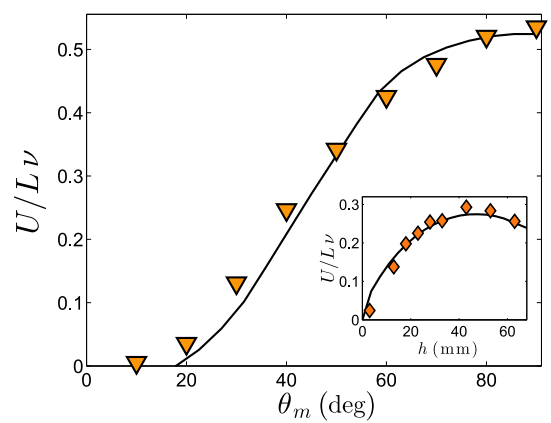

FIG. 2. Mean speed of the rod's head along the $x$-axis as a function of the experimental parameters: angular velocity $L v\left(\right.$ a), angular amplitude $\theta_{m}$ (b), and depth $h$ (inset). Other parameters are maintained constants: $L=60 \mathrm{~mm}, D=6 \mathrm{~mm}, v=0.26 \mathrm{~Hz}, h=40 \mathrm{~mm}, \theta_{m}=45^{\circ}$. In (b) the solid lines show the predictions of the numerical simulation introduced in Sec. III for $\alpha=0.28, \theta_{t}=8^{\circ}, \theta_{i}=40^{\circ}, C_{n} / C_{t}=3$, and $F_{d} / C_{n} L=$ $0.055 \mathrm{~h} / \mathrm{L}$.

\section{A. Dynamics}

We first explore the behavior of the oscillating rod in pure glycerine (density $\rho_{f}=1260 \mathrm{~kg} / \mathrm{m}^{3}$ and dynamic viscosity $\eta_{f}=1.41 \mathrm{~Pa}$ ), which ensures Reynolds numbers below $2 \times 10^{-2}$. The rod head experiences a back and forth motion without drift as predicted by Purcell's scallop theorem for a Newtonian fluid [blue solid line in Fig. 1(b)]. In the granular medium, the same back-and-forth motion of the rod head is observed, but it is superposed to a large drift along the $x$-direction of about $80 \%$ of the stroke [orange solid line in Fig. 1(b)]. One notes that the direction of the drift is opposed to the one of a tail oscillating in a fluid at large Reynolds numbers. These observations reveal that the propulsion in granular media occurs very differently from that in Newtonian fluids. We checked that the substitution of the sinusoidal motion of the rod by a triangular motion does not affect its propulsion. Moreover, the initial packing fraction of the granular was varied from $\phi=0.58$ to $\phi=0.61$, and a drift of the system was observed independently of the initial packing fraction (see Appendix A). Thus, we conclude that the present phenomenon is the result of a generic property of particulate materials. Thereafter, we measured the mean speed $U$ of its head along the $x$-axis as a function of the angular velocity and amplitude [Figs. 2(a) and 2(b)]. The linearity between $U$ and $L v$ reveals the absence of inertia in our experiments as expected by the low value of the granular inertial number $\left(I=d \dot{\gamma} / \sqrt{P / \rho_{g}}<0.1\right.$ where $\dot{\gamma}$ is the shear rate of the granular flow around the object estimated as $L v / D, \rho_{g}$ the grain density, and $P$ the confining pressure that is in our case $\rho g h$ and results from the weight of the granular column) [19]. At a given frequency, the mean velocity of the oscillating rod normalized by $L v$ is observed to increase nonlinearly with $\theta_{m}$ [Fig. 2(b)]. Furthermore, we explored the influence of the depth $h$ of the rod below the granular surface on its motion and reported an optimal depth that maximizes $U$ [Fig. 2(b), inset].

\section{B. Torque measurement}

We use a torque sensor to measure the vertical torque $T$, necessary to sustain rod rotation and provide insight into the origin of this propulsion. For a rod moving in a reciprocal motion into a viscous fluid at low Reynolds numbers, the torque cycle is symmetric about the central axis $(\theta=0)$ (Fig. 3, dashed lines). In a granular medium, the situation strongly differs from viscous fluids (Fig. 3, dark solid line), and three specific features are observed: a drop of the torque after the first stroke, a transient regime after a change of the moving direction, and a torque increase at the end of the stroke. 


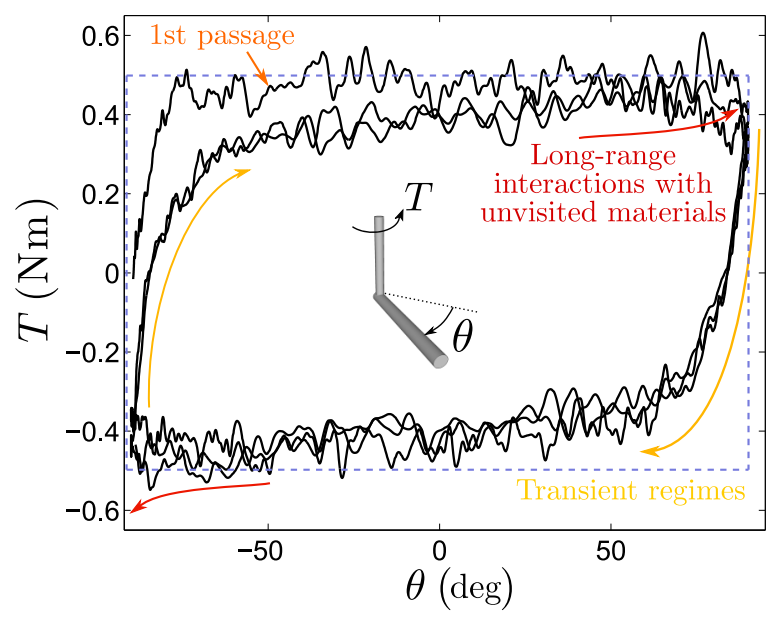

FIG. 3. Torque $T$ experienced by a $\operatorname{rod}(L=60 \mathrm{~mm}$ and $D=6 \mathrm{~mm})$ in a granular medium as a function of its angular position for a constant depth $h=40 \mathrm{~mm}$ and $\theta_{m}=90^{\circ}$. The blue dashed line sketches the torque for the same cycle in a viscous fluid.

First, the torque measurements reveal that the torque (or equivalently the drag force defined as $F=2 T / L$ ) is larger for the first passage of the rod (indicated by the orange arrow in Fig. 3) than for the following passages. We determine that the mean torque value for the first passage is $28 \%$ larger than the mean value measured for subsequent passages. A similar reduction of the drag force has been observed by Guillard et al. for a cylinder that rotates continuously in a tank of glass beads [20]. In their experiment, the drag reduction occurs after the first half-rotation and reaches a depth-independent regime after a full rotation. This effect was attributed to a redirection of the principal stress away from the object after its first passage and to the creation of a bubble of low pressure screening the hydrostatic pressure arising from the column of material placed above the object [21]. In the following, the reduction of the drag force will be referred as a "structuring effect" as it results from a change in the microstructure of the medium after the passage of the object.

Second, we observe that right after a rotation inversion, the torque displays a transient regime (indicated by the yellow arrows in Fig. 3) before recovering its nominal value $T_{0}=T(\theta=0)$. This contrasts with the viscous drag that establishes over a null distance in the limit of zero Reynolds numbers. We measure the transient torque after rod inversion for various rod lengths $L$ [Fig. 4(a)]. These measurements are well adjusted by an exponential law $T \sim T_{0}\left(1-e^{-\theta / \theta_{t}}\right)$ with $\theta_{t}=8 \pm 1^{\circ}$. The characteristic angle $\theta_{t}$ is observed not to depend on $L$ but rather to be a characteristic of the granular medium.

Third, the torque cycle shows that when the rod approaches an "unvisited region." i.e., a zone it has never visited before, the associated drag gradually increases to the value observed during the first passage of the rod (indicated by the red arrows in Fig. 3). This effect explains the gradual increase of the torque as the rod approaches the course extremes and is attributed to long-range interactions between the rod and the undisturbed microstructure of the "unvisited region." We measure that the torque recovers its first value within a precision of $10 \%$ on a characteristic angle $\theta_{i} \simeq 40 \pm 5^{\circ}$.

\section{Consequences for propulsion}

These three phenomena together break the inversion symmetry of the system and contribute to explain the observed drift of the oscillating rod. Indeed, during propulsion along the $x$-direction, a segment of the rod is always visiting new zones of the granular medium. Figure 5(a) shows the superposition of rod's positions over time and highlights in color the new zones visited during a quarter of a cycle. The drag is larger when the rod moves across unvisited zones [orange in Fig. 5(a)], 
(a)

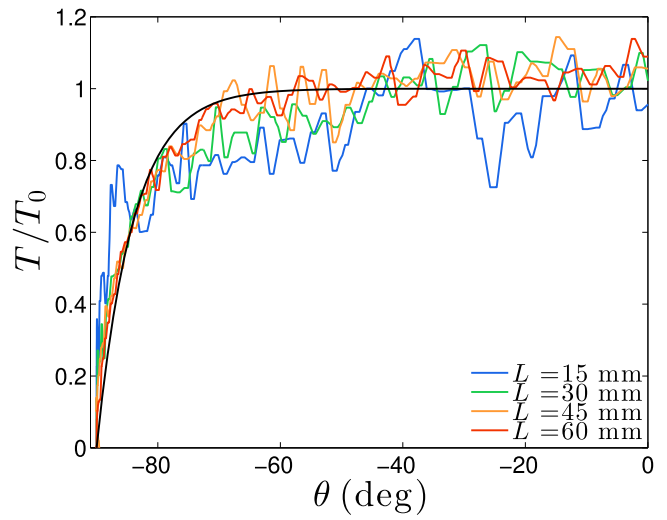

(b)

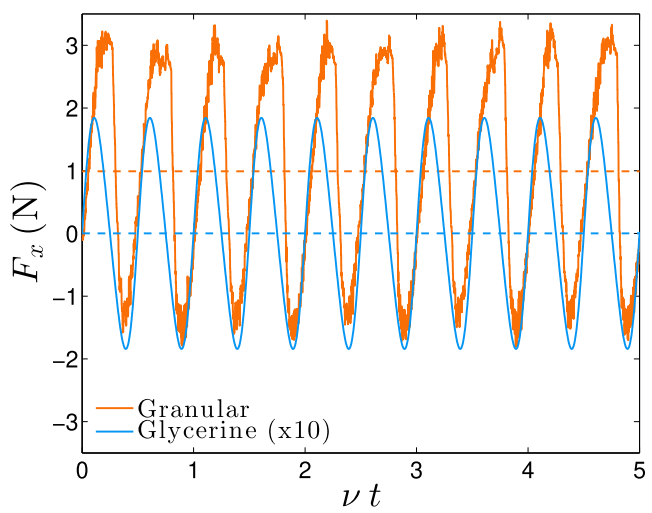

FIG. 4. (a) Normalized torque $T / T_{0}$ experienced by a rod as a function of its angular position $\theta$ after a change in direction $(D=6 \mathrm{~mm}$ and $h=40 \mathrm{~mm})$. The blue, green, orange, and red solid lines correspond to rod lengths $L=15,30,45,60 \mathrm{~mm}$, respectively. The dark solid line indicates the empirical relation $T=$ $T_{0}\left(1-e^{-(\theta+90) / \theta_{t}}\right)$ with $\theta_{t}=8^{\circ}$. (b) Force along the $x$-axis as a function of the normalized time for a rod with a length of $L=60 \mathrm{~mm}$ and diameter of $D=6 \mathrm{~mm}$ oscillating at $v=0.26 \mathrm{~Hz}$ with an angular amplitude $\theta_{m}=90^{\circ}$ into a granular medium at a depth $h=40 \mathrm{~mm}$ (orange solid line). The theoretical force experienced by the same rod moving in glycerine is indicated by the blue solid line as a reference and magnified by a factor of 10 .

which produces forward steps that are larger than backward steps. Moreover, at the end of it course, the rod interacts with "unvisited" zones through long-range interactions [red in Fig. 5(a)], which increases its drag and its forwards motion. After the maximal angle $\left(\theta= \pm \theta_{m}\right)$ is reached (rotation inversion) the rod undergoes a transient regime and mainly interacts with "visited" zones that both result in a lower drag [yellow in Fig. 5(a)]. These two effects favor the forward direction. The combination of these three phenomena (structuring effect, transient after inversion, and long-range interactions with unvisited material) on the force $F_{x}$ generated by the rod along the $x$-direction is presented in Fig. 4(b). One notes the strong asymmetry $(\sim 46 \%)$ of the projected force $F_{x}$ between forwards and backwards motions that is at the origin of the rod net displacement in granular media that contrasts with the symmetric force experienced in a viscous fluid.

(a)

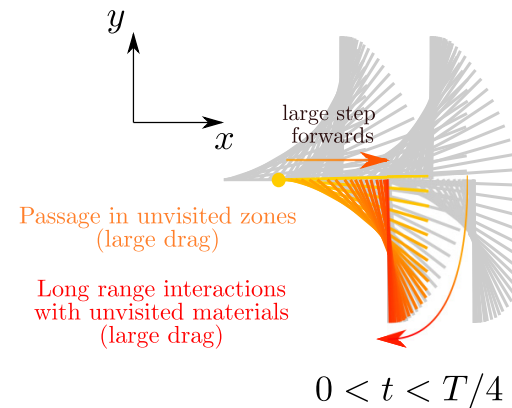

(b)

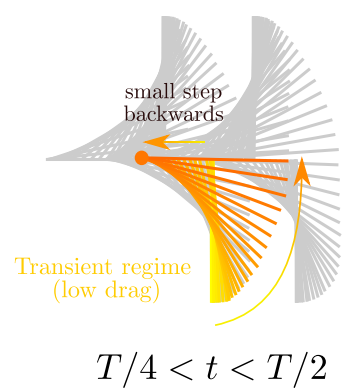

FIG. 5. Successive positions of the rod and illustrations of the three mechanisms that govern its propulsion into a granular medium. A quarter of the cycle is highlighted in color. Color codes the norm of the drag: decreasing drag from red to yellow. Arrows indicate the sense of rod motion. (a) The head moves forward while the rod explores mainly unvisited zones. (b) The head moves backward in a less extend while the rod explores unvisited zones. 


\section{MODEL}

In order to model the features of the observed propulsion, the dynamical equations for the rod are considered along with the generalized Coulomb's friction law in which the friction force $f$ of a rod segment is expressed in normal and tangential components to the rod [ $\boldsymbol{e}_{r}$ and $\boldsymbol{e}_{\theta}$ as defined in Fig. 1(a)], $\boldsymbol{f}=-C_{n}\left(\boldsymbol{e}_{v} \cdot \boldsymbol{e}_{\theta}\right) \boldsymbol{e}_{\theta}-C_{t}\left(\boldsymbol{e}_{v} \cdot \boldsymbol{e}_{r}\right) \boldsymbol{e}_{r}$ where $\boldsymbol{e}_{v}$ is the unit vector associated to the velocity $v$ of the rod segment [22]. The coefficients $C_{n}$ and $C_{t}$, associated with the normal and tangential directions, respectively, correspond to a force per unit length and have been shown to be proportional to the granular pressure at the object location $\rho g h$ and the perimeter of the slender body cross section $\pi D[2,20]$. For our system, we determine experimentally these friction coefficients through independent drag measurements in a stationary regime and obtain $C_{n} \simeq 13 \mathrm{D} \rho \mathrm{gh}$ and $C_{n} / C_{t} \simeq 3$. In a quasistatic limit, the sum of the projection of the forces experienced by the rod segments along the $x$-direction, $F_{x}=\int_{0}^{L} \boldsymbol{f} \cdot \boldsymbol{e}_{x} d r$, is balanced by the total drag experienced by the system, $F_{d}$. In practice, this drag mainly results from the resistance of the vertical rod that transmits the motor rotation. Thus, the dynamics of the rod is ruled by the relation

$$
\operatorname{sgn}\left(v_{x}\right) \int_{0}^{L} \frac{+C_{n}\left(r \dot{\theta}-v_{x} \sin \theta\right) \sin \theta-C_{t} v_{x} \cos ^{2} \theta}{\sqrt{\left(v_{x}-r \dot{\theta} \sin \theta\right)^{2}+(r \dot{\theta} \cos \theta)^{2}}} d r=F_{d},
$$

where $v_{x}$ is the head velocity along the $x$-direction and the unknown of the problem. Equation (1) can be solved analytically in the ideal case where the drag force does not depend on the history of the rod motion (see Appendix B). However, our previous observations have shown that this assumption does not hold and that a history-dependent model of the friction force is required.

In order to account for the features of the drag force described previously (see Fig. 3), we consider the plastic response of the granular medium to the shear deformation. We assume that the elasticity of the material is at play and the total deformation, $u$, is the sum of the elastic deformation $\epsilon$ and the plastic deformation $v: u=\epsilon+v$. The shear stress is then $\sigma=\epsilon / G$, where $G$ is the shear modulus of the granular compact. The plastic process activates above a yield stress, $\sigma_{Y}$, and the shear stress saturates to the maximum value, $\sigma_{0}$, that the material can sustain. The simplest functional form for the elementary plastic deformation is $d v=\left(\sigma-\sigma_{Y}\right) / \sigma_{0} d u$ [23]. Combining these equations, we get $\sigma=\sigma_{Y}+\sigma_{0}\left(1-e^{-G\left(u-\epsilon_{Y}\right) / \sigma_{0}}\right)$ for $u>\epsilon_{Y}$ with $\epsilon_{Y}=\sigma_{Y} / G$. This stress-displacement relation has been verified with foam under cycle loading [23]. In granular media, an exponential relaxation of the stress has also been reported [24] but with a characteristic deformation that is much larger than the prediction, $\sigma_{0} / G$, if we estimate $G$ with Walton's formula [25]. This difference reveals that the observed response cannot be related solely to material elasticity and that a better description of elastic and plastic events in cyclic loading should be considered [26]. Nonetheless, our approach provides a basic model to rationalize the modification of the granular drag experienced by an object as it moves. Indeed, the previous stress-deformation relation is directly equivalent to our torque-angle relation when transposed into circular coordinates through $d \theta \simeq 2 D / L d u$ and $T \simeq \sigma D L^{2} / 2$. In this model, the three phenomena described previously in the history of the drag force (structuring effect, the transient regime after inversion, and long-range interactions, respectively) can be interpreted as a decrease of the shear modulus $G$ and the maximal shear stress $\sigma_{0}$ after the passage of the object into an "unvisited" zone. This effect could result from a modification of the material microstructure, either packing fraction or force network, and remains to be investigated. Here we adopt a semiempirical approach where the forces follow the exponential relaxation predicted previously and heuristic rules to account for the change of material properties. Finally, we consider that the drag force respects the following rules:

(1) A gradual relaxation of the friction force after each rotation inversion according to an exponential law $f=f_{0}\left(1-e^{-\theta / \theta_{t}}\right)$ with $f_{0}$ the nominal force per unit length for the first passage.

(2) A drop of the friction force by a factor $\alpha\left(f=(1-\alpha) f_{0}\right)$ when moving through materials already visited.

(3) A recovery of the friction force towards its nominal value ( $\left.f=f_{0}\right)$ when the rod is coming close to unvisited material $\left(|\theta|>\theta_{i}\right.$ and $\left.\theta \dot{\theta}>0\right)$. 
(a)

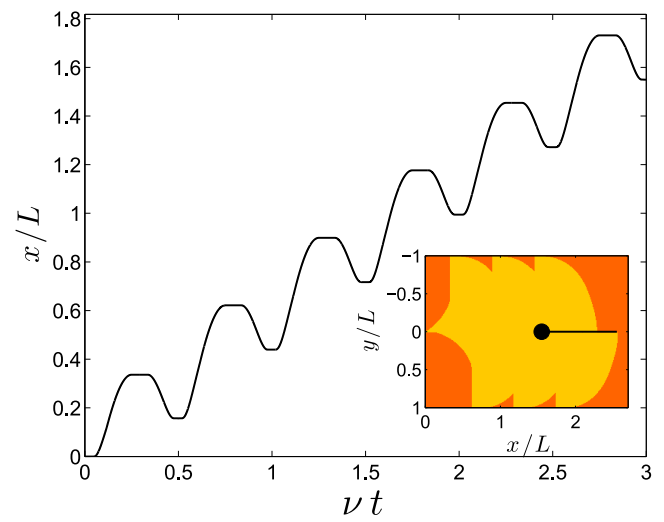

(b)

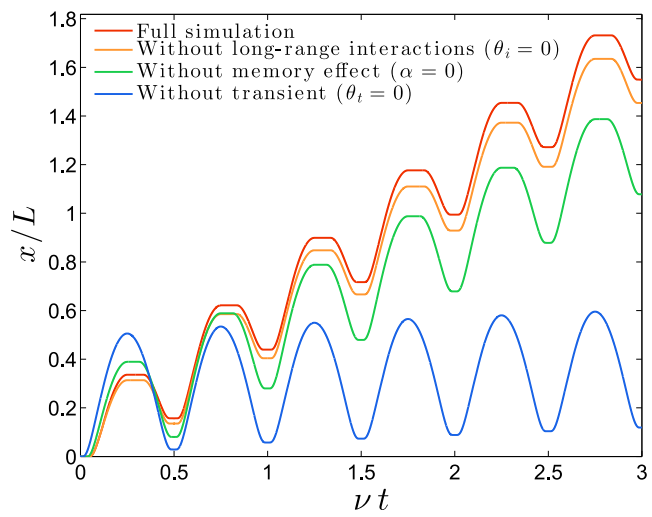

FIG. 6. (a) Normalized displacement of the rod's head, $x / L$, as a function of $v t$ resulting from the numerical integration of Eq. (1) with the three rules to account for structuring effects, transient regimes, and long-range interactions. The parameters are $\theta_{m}=90^{\circ}, C_{n} / C_{t}=3, F_{d} / L C_{n}=0.08, \alpha=0.28, \theta_{t}=8^{\circ}$, and $\theta_{i}=40^{\circ}$. Inset: visited (yellow) and nonvisited (orange) areas in the $(x, y)$ plane after three oscillations of the rod (black solid line). (b) Normalized head position $x / L$ as a function of the normalized time $v t$. The red solid line corresponds to the numerical integration when the three effects are taken into account, the orange solid line in the absence of long-range interactions $\left(\theta_{i}=0^{\circ}\right)$, the green solid line in the absence of memory effect $(\alpha=0)$, and the blue solid line in the absence of transient regime $\left(\theta_{t}=0^{\circ}\right)$.

These empirical rules involve three parameters $\alpha, \theta_{t}$ and $\theta_{i}$ that can be determined experimentally through torque measurements. When Eq. (1) is coupled with these rules, no simple solution can be found, and we solved the system numerically to predict the rod dynamics. We consider the parameters determined experimentally for our system through independent drag measurements $\left(C_{n} / C_{t}=3, F_{d} / L C_{n}=0.055 h / L\right)$ and torque measurements $\left(\alpha=0.28, \theta_{t}=8^{\circ}\right.$, and $\left.\theta_{i}=40^{\circ}\right)$. Figure 6(a) shows the numerical solution of the normalized displacement of the rod's head $x / L$ as a function of the normalized time $v t$. Moreover, the inset in Fig. 6(a) presents the zones visited by the rod after three oscillations. These results clearly show that the rod propels along the $x$-direction and visits new material with every strokes. Numerical solutions of the model also predict the normalized mean speed of the rod's head $U / L v$ as a function of $\theta_{m}$ [Fig. 2(b), dark solid line] and $h$ [inset in Fig. 2(b), dark solid line]. These predictions are in good agreement with experiments supporting the proposed empirical rules.

Thereafter, we use the numerical solution of this problem to inspect the contribution of each phenomenon to the global propulsion. By turning off the transient regime after an inversion (setting $\theta_{t}=0^{\circ}$ ), we showed that the structuring effect and long-range interactions alone are not sufficient to induce a permanent drift of the rod [Fig. 6(b)]. However, it is observed that the propulsion of the rod is enhanced by the presence of these two phenomena. We conclude that the asymmetry of torque cycle, with respect to the $x$-axis, is responsible for propulsion.

\section{PERSPECTIVES AND CONCLUSION}

The three phenomena responsible for the displacement of an oscillating rod in a granular medium all have important consequences for how animals and robots interact with such materials. In fact, including these phenomena may either enhance or diminish propulsion based on nonreciprocal motion in a granular system [9,27]. Taking these effects into account to model problems of locomotion in granular media should contribute to the development of better sand-swimming robots with promising applications in rescue and exploration missions [3]. In nature, the darkling beetle 
has developed strategies of locomotion which take advantage of propulsive forces in granular matter through the use of its legs in a coordinated and reciprocal motion. Both legs oscillate in phase opposition and perform a cycle similar to the one described here. However, further investigation is needed to fully elucidate the role of legs geometry, in particular the breaking of the forwardbackward symmetry.

Finally, we investigate the generality of this mode of propulsion for materials that differ from dry granular media. We immersed our system into different non-Newtonian liquids and studied the corresponding dynamics of the oscillating rod. Three different fluids were tested: a dense suspension of cornstarch (55\% in weight) which is shear-thickening [28], a bentonite solution ( $8 \%$ in weight) which is thixotropic [29], and a dry-liquid foam (gas volume fraction $\phi=96 \%$ ) which can be approached as a yield-stress fluid. We observed a large propulsion of the rod in a cornstarch solution for high rotation speeds (drift of $30 \%$ of the stroke), a small drift in bentonite dispersions (5\% of the stroke), and a negligible drift in foam (below the experimental resolution). These observations lead to the conclusion that the mechanism responsible for the net displacement of an object undergoing a reciprocal and time-reversible motion is the change of the material properties within the solicitation time, which mimics a transient regime. As many biological and industrial fluids have time-dependent properties, propulsion strategies based on reciprocal and reversible motions would operate in these contexts.

We conclude that unlike viscous fluids, reciprocal motions may induce a net motion of a single freedom-degree propeller inside granular materials. We identified three distinct phenomena that contribute to this propulsion: a structuring effect operating on the drag force experienced by the object that is lower after the first passage, a transient regime affecting the nominal drag force that is attained after a given traveled distance, and a long-range interaction revealed by the fact that the drag is larger when approaching unvisited regions of the granular sample. These observations compel us to refine the accepted physical description of the drag of an object in a granular material. The rationalization of these mechanisms opens opportunities to design small robots able to propel in granular media and control food storage in silos or perform medical investigations into complex biological fluids.

\section{ACKNOWLEDGMENTS}

We acknowledge the support from Dirección de Investigación Científica y Tecnológica (DICYT) of Universidad de Santiago for the Project No. 041731MH-POSTDOC. The Laboratoire International Associé "Matière: Structure et Dynamique" (LIA-MSD) is kindly acknowledged for encouraging this scientific research. F.M. acknowledges ANID-Chile through Fondecyt Project No. 1201013 and Fondequip 130149.

\section{APPENDIX A: INFLUENCE OF PACKING FRACTION}

This Appendix considers the effect of the packing fraction on the drag experienced by an oscillating rod. Figure 7 displays the torque $T$ experienced by the rod oscillating at the same place for two different preparations of the granular medium (packing fraction of $\phi=0.58$ and $\phi=0.61$ ). We observed that for the first cycle, the torque exerted on the rod is larger for the granular of higher packing fraction. However, after the first cycle, the differences between packing fraction fades and the torque reach a value that does not depend on the initial preparation. This observation explains the fact of our observing the propulsion of an oscillating rod whatever the initial preparation of the granular material.

\section{APPENDIX B: ANALYTICAL DEVELOPMENT}

In this Appendix we solve the dynamics of a rod oscillating horizontally within a granular medium. The angle made by the rod with the axis $x$ is denoted $\theta$. Two unit vectors are introduced to account for the rod position and orientation: a tangential $\boldsymbol{e}_{r}=\cos \theta \boldsymbol{e}_{x}+\sin \theta \boldsymbol{e}_{y}$ and 


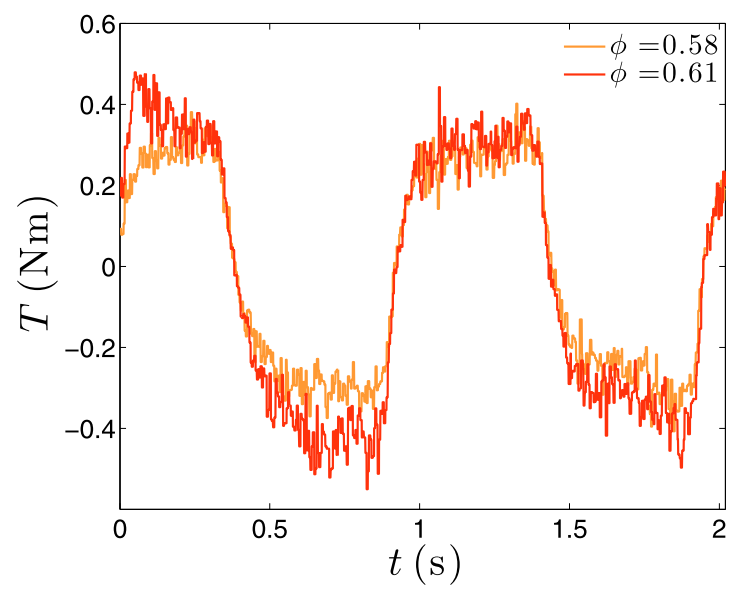

FIG. 7. Torque $T$ experienced by the rod as a function of time for two different packing fractions of the granular medium $\phi=0.58$ (orange solid line) and $\phi=0.61$ (red solid line).

a normal $\boldsymbol{e}_{\theta}=-\sin \theta \boldsymbol{e}_{x}+\cos \theta \boldsymbol{e}_{y}$ (Fig. 8). The velocity of the point $\mathrm{O}$ (the head) along the $x$-direction is denoted $v_{x}$, and the velocity of a segment of the rod located at a distance $r$ is written $\boldsymbol{v}=v_{x} \boldsymbol{e}_{x}+r \dot{\theta} \boldsymbol{e}_{\theta}$ and is associated to the unit vector $\boldsymbol{e}_{v}$. Thus, the velocity of a segment in the $\left(\boldsymbol{e}_{r}, \boldsymbol{e}_{\theta}\right)$ frame is $v_{r}=\boldsymbol{v} \cdot \boldsymbol{e}_{r}=v_{x} \cos \theta$ and $v_{\theta}=\boldsymbol{v} \cdot \boldsymbol{e}_{\theta}=r \dot{\theta}-v_{x} \sin \theta$. The force per unit length $\boldsymbol{f}=-C_{n}\left(\boldsymbol{e}_{v} \cdot \boldsymbol{e}_{\theta}\right) \boldsymbol{e}_{\theta}-C_{t}\left(\boldsymbol{e}_{v} \cdot \boldsymbol{e}_{r}\right) \boldsymbol{e}_{r}$ applied to a segment located in $(r, \theta)$ is written

$$
\boldsymbol{f}=\frac{-C_{n}\left(r \dot{\theta}-v_{x} \sin \theta\right) \boldsymbol{e}_{\theta}-C_{t} v_{x} \cos \theta \boldsymbol{e}_{r}}{\sqrt{\left(v_{x}-r \dot{\theta} \sin \theta\right)^{2}+(r \dot{\theta} \cos \theta)^{2}}} .
$$

In a steady regime, the sum of the projection of the forces along the $x$-direction, $F_{x}=\int_{0}^{L} \boldsymbol{f} \cdot \boldsymbol{e}_{x} d r$ is balanced by the total drag experienced by the system, $F_{d}$. Thus, the dynamics of the rod is ruled by the relation

$$
\operatorname{sgn}\left(v_{x}\right) F_{d}=\int_{0}^{L} \frac{+C_{n}\left(r \dot{\theta}-v_{x} \sin \theta\right) \sin \theta-C_{t} v_{x} \cos ^{2} \theta}{\sqrt{\left(v_{x}-r \dot{\theta} \sin \theta\right)^{2}+(r \dot{\theta} \cos \theta)^{2}}} d r .
$$

We normalized Eq. (B2) with $\bar{r}=r / L, \bar{v}=v_{x} / L v, \bar{\theta}=\dot{\theta} / v$, and $\bar{F}_{d}=F_{d} / L C_{n}$ and found

$$
\operatorname{sgn}\left(v_{x}\right) \bar{F}_{d}=\int_{0}^{1} \tilde{f} \frac{(\bar{r} \bar{\theta}-\bar{v} \sin \theta) \sin \theta-\frac{C_{t}}{C_{n}} \bar{v} \cos ^{2} \theta}{\sqrt{\bar{v}^{2}+(\bar{r} \dot{\theta})^{2}-2 \bar{v} \bar{r} \dot{\theta} \sin \theta}} d \bar{r}
$$

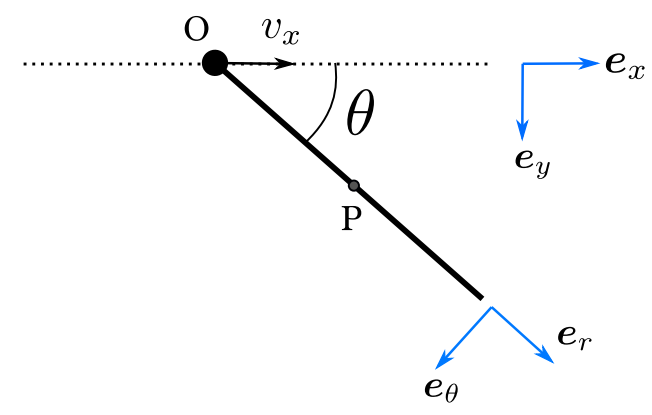

FIG. 8. Notations used in the model 
where $\tilde{f}=f / f_{0}$ is the ratio of the force over its nominal value. In the general case, the normalized friction force $\tilde{f}$ depends on the local history of the medium and varies with time and space. Equation (B3) can be solved in the specific case where the friction force is homogeneous and constant $(\tilde{f}=1)$ and where tangential friction is negligible $\left(C_{t} / C_{n}=0\right)$. Such a situation may occur during the first step of the rod within the granular medium. These assumptions lead to

$$
\bar{F}_{d}=\sin \theta \int_{0}^{1} \frac{\bar{r} \dot{\dot{\theta}}-\bar{v} \sin \theta}{\sqrt{\bar{v}^{2}+(\bar{r} \dot{\dot{\theta}})^{2}-2 \bar{v} \bar{r} \dot{\theta} \sin \theta}} d \bar{r}
$$

which transforms into

$$
\frac{\overline{\dot{\theta}} \bar{F}_{d}}{\sin \theta}=\sqrt{\overline{\dot{\theta}}+\bar{v}^{2}-2 \bar{v} \sin \theta}-\bar{v} .
$$

This equation has physical solutions only for $\sin \theta>\bar{F}_{d}$ meaning that the propulsive force overcomes the resistive force and allows the system to move along the $x$-direction. In this case, the normalized velocity is written

$$
\bar{v}=\overline{\dot{\theta}} \frac{\sin \theta-\bar{F}_{d}}{2\left(\sin ^{2} \theta+\bar{F}\right)} .
$$

Equation (B6) provides the position of the point $\mathrm{O}$ as a function of $\theta: \bar{x}_{O}=\int_{0}^{\bar{t}} \bar{v} d \bar{t}$. Moreover, the position $\left(\bar{r}_{p}, \theta\right)$ of the pivoting point $P$ derives from the relation $\bar{r}_{p} \sin \theta=\bar{v}$. The tip of the $\operatorname{rod}$ follows a curve determined by the relation $d \bar{s} / d \theta=1-\bar{r}_{p}$. Apart from these approximations on the normalized force $\tilde{f}$ and the ratio $C_{t} / C_{n}$, Eq. (B3) has no simple analytical solution and has to be solved numerically.

[1] W. Baumgartner, F. Fidler, A. Weth, M. Habbecke, P. Jakob, C. Butenweg, and W. Böhme, Investigating the locomotion of the sandfish in desert sand using NMR-imaging, PloS ONE 3, e3309 (2008).

[2] R. D. Maladen, Y. Ding, C. Li, and D. I. Goldman, Undulatory swimming in sand: Subsurface locomotion of the sandfish lizard, Science 325, 314 (2009).

[3] R. D. Maladen, Y. Ding, P. B. Umbanhowar, and D. I. Goldman, Undulatory swimming in sand: Experimental and simulation studies of a robotic sandfish, Int. J. Robot. Res. 30, 793 (2011).

[4] D. Goldman, H. Komsuoglu, and D. Koditschek, March of the sandbots, IEEE Spectrum 46, 30 (2009).

[5] C. Koch, Some aspects of abundant life in the vegetationless sand at the Namib Desert dunes, Sci. Papers Namib Desert Res. Station 1961, 9 (1961).

[6] E. Holm and E. B. Edney, Daily activity of Namib Desert arthropods in relation to climate, Ecology 54, 45 (1973).

[7] G. N. Louw, S. W. Nicolson, and M. K. Seely, Respiration beneath desert sand: Carbon dioxide diffusion and respiratory patterns in a tenebrionid beetle, J. Exp. Biol. 120, 443 (1986).

[8] J. Lighthill, Flagellar hydrodynamics, SIAM Rev. 18, 161 (1976).

[9] R. D. Maladen, Y. Ding, P. B. Umbanhowar, A. Kamor, and D. I. Goldman, Mechanical models of sandfish locomotion reveal principles of high performance subsurface sand-swimming, J. R. Soc., Interface 8, 1332 (2011).

[10] C. Li, T. Zhang, and D. I. Goldman, A terradynamics of legged locomotion on granular media, Science 339, 1408 (2013).

[11] T. Zhang and D. I. Goldman, The effectiveness of resistive force theory in granular locomotion, Phys. Fluids 26, 101308 (2014).

[12] H. Askari and K. Kamrin, Intrusion rheology in grains and other flowable materials, Nat. Mater. 15, 1274 (2016). 
[13] E. M. Purcell, Life at low Reynolds number, Am. J. Phys. 45, 3 (1977).

[14] E. Lauga, Life around the scallop theorem, Soft Matter 7, 3060 (2011).

[15] O. S. Pak, T. Normand, and E. Lauga, Pumping by flapping in a viscoelastic fluid, Phys. Rev. E 81, 036312 (2010).

[16] T. Qiu, T.-C. Lee, A. G. Mark, K. I. Morozov, R. Münster, O. Mierka, S. Turek, A. M. Leshansky, and P. Fischer, Swimming by reciprocal motion at low Reynolds number, Nat. Commun. 5, 5119 (2014).

[17] E. Lauga and D. Bartolo, No many-scallop theorem: Collective locomotion of reciprocal swimmers, Phys. Rev. E 78, 030901(R) (2008).

[18] A. Seguin, Y. Bertho, and P. Gondret, Influence of confinement on granular penetration by impact, Phys. Rev. E 78, 010301(R) (2008).

[19] Y. Forterre and O. Pouliquen, Flows of dense granular media, Annu. Rev. Fluid Mech. 40, 1 (2008).

[20] F. Guillard, Y. Forterre, and O. Pouliquen, Depth-Independent Drag Force Induced by Stirring in Granular Mediac, Phys. Rev. Lett. 110, 138303 (2013).

[21] F. Guillard, Y. Forterre, and O. Pouliquen, Origin of a depth-independent drag force induced by stirring in granular media, Phys. Rev. E 91, 022201 (2015).

[22] B. Darbois Texier, A. Ibarra, and F. Melo, Helical Locomotion in a Granular Medium, Phys. Rev. Lett. 119, 068003 (2017).

[23] J.-C. Géminard, J. C. Pastenes, and F. Melo, Foam rheology at large deformation, Phys. Rev. E 97, 042601 (2018).

[24] A. Seguin, Hysteresis of the drag force of an intruder moving into a granular medium, Eur. Phys. J. E 42, 13 (2019).

[25] K. Walton, The effective elastic moduli of a random packing of spheres, J. Mech. Phys. Solids 35, 213 (1987).

[26] J. Boschan, S. Luding, and B. P. Tighe, Jamming and irreversibility, Granular Matter 21, 58 (2019).

[27] Z. Peng, Y. Ding, K. Pietrzyk, G. J. Elfring, and O. S. Pak, Propulsion via flexible flapping in granular media, Phys. Rev. E 96, 012907 (2017).

[28] E. E. B. White, M. Chellamuthu, and J. P. Rothstein, Extensional rheology of a shear-thickening cornstarch and water suspension, Rheol. Acta 49, 119 (2010).

[29] P. C. F. Møller, J. Mewis, and D. Bonn, Yield stress and thixotropy: On the difficulty of measuring yield stresses in practice, Soft Matter 2, 274 (2006). 